\title{
Accuracy of blood pressure monitors available in high street pharmacies
}

Running head: Accuracy of consumer BP monitors

Authors: Adam Ware ${ }^{2}$, Richard Stevens ${ }^{1}$, Mary Selwood ${ }^{1}$, Susannah Fleming ${ }^{1}$

${ }^{1}$ Nuffield Department of Primary Care Health Sciences, University of Oxford

${ }^{2}$ Keble College, University of Oxford

\section{Corresponding author:}

Susannah Fleming

Nuffield Department of Primary Care Health Sciences

Radcliffe Observatory Quarter

Woodstock Road

Oxford OX2 6GG

UK

Tel: +44 (0) 1865289220

susannah.fleming@phc.ox.ac.uk

Requests for reprints should be addressed to the corresponding author.

Conflicts of Interest and Source of Funding: The study was funded through a fellowship held by Dr Fleming, funded by the National Institute of Health Research (NIHR) School for Primary Care Research. The funder had no input into the design of the study, collection or analysis of data, or the decision to publish. Mr Ware, Dr Stevens, and Ms Selwood declare no potential conflict of interest. The views expressed in this paper are those of the authors and not necessarily those of the NHS, the NIHR, or the Department of Health. 


\section{Abstract \\ Objective}

To assess the accuracy of automated blood pressure monitors on sale to the UK general public.

\section{Methods}

We conducted static pressure accuracy testing on all compatible (19 of 22 available) blood pressure monitors available for sale in pharmacies within the city of Oxford, UK, and tested two devices for accuracy in measurement of systolic and diastolic blood pressure in 21 adults.

\section{Results}

Devices showed good accuracy when measuring static pressure in laboratory bench testing with the median error per device ranging from $-2.2 \mathrm{mmHg}$ to $+1.2 \mathrm{mmHg}$; however the two devices tested in vivo performed worse than in laboratory tests, with median errors as high as $6 \mathrm{mmHg}$.

\section{Conclusions}

Monitors showed good accuracy in static pressure testing, with a lack of correlation between monitor price and accuracy. However, higher errors seen during in vivo testing of a subset of monitors may indicate that static testing may not be appropriate for routine accuracy assessment of these monitors.

\section{Keywords}

Sphygmomanometers, pharmacies, accuracy 


\section{Introduction}

The majority of automated blood pressure monitors on sale to the public have been clinically validated; they have passed at least one of the recognised accuracy protocols.[1-3] However, a systematic review of published validation studies found that devices could pass these protocols with as few as $60 \%$ of measurements within $5 \mathrm{mmHg}$ of the true blood pressure value.[4] Monitors used in GPs surgeries have shown considerable variance in accuracy.[5] Our study aimed to test the accuracy of monitors marketed to the general public for home measurement of blood pressure.

\section{Methods}

Monitors for testing were selected by purchasing all available models from pharmacies with the city of Oxford, UK (postcodes OX1-OX4). These were tested for accuracy in measuring static pressures using the Omron PA-350, a calibrated reference device designed for testing the accuracy of blood pressure monitors. The Omron PA-350 was used to generate static pressure values at $20 \mathrm{mmHg}$ increments between 50 and $250 \mathrm{mmHg}$ with the reference (Omron PA-350) and test device (blood pressure monitor) readings being recorded at each point. A total of 10 readings at each of these pressure ranges were made for each monitor, with half of the readings being in each of increasing and decreasing pressure regimens, giving a total of 110 readings per monitor. This process is similar to typical calibration procedures for blood pressure monitors, but with an increased number of measurements, and fulfils the requirements for pressure transducer accuracy tests in the AAMI standard.[2]

Two monitors were selected from those with static pressure results for further testing on 21 adult human volunteers. Monitors were selected without knowledge of the results of static testing, and were chosen to represent both higher and lower cost monitors. This aspect of the study was approved by the University of Oxford Medical Sciences Ethics Committee (MSD/IDREC/2011/9), and written informed consent was obtained from all participants. We 
followed the ESH-IP protocol for this study, with deviations as specified below. We used the Homedics BPA-300 digital monitor as the reference monitor, as it was known to have passed the AAMI validation protocol with a high percentage of both systolic and diastolic readings within $5 \mathrm{mmHg}$ of the reference value,[4] and we did not have access to suitably experienced personnel to carry out accurate measurements by auscultation with a mercury

sphygmomanometer. As we were testing two monitors, we used one on the left arm, and one on the right arm for each subject, with test monitors being alternated between arms for each subsequent subject. We took measurements on the left arm first, following the order from ESHIP,[3] with seven blood pressure readings being taken beginning with the reference monitor and alternating with the test monitor. This procedure was then repeated on the right arm with the other test monitor. If a device reported an error message, the measurement was repeated a maximum of two further times in an attempt to get a reading. These deviations, and the smaller sample size used, mean that this preliminary study is not equivalent to a validation study.

\section{Results}

Of 22 monitors purchased, 19 were compatible with the Omron PA-350, and were successfully tested for accuracy in measurement of static pressures. The mean absolute error across the devices was $0.672 \mathrm{mmHg}$. Fig. 1 shows a box plot of the errors for the tested monitors, in descending order of retail price. As can be seen from the figure, there is little correlation between retail price and monitor accuracy. Median errors ranged from $0 \mathrm{mmHg}$ for the most accurate device to $-2.2 \mathrm{mmHg}$ for the least accurate, where negative numbers indicate that the test device underestimates the reference pressure.

Tested monitors demonstrate both positive and negative bias in the median error, corresponding to under- or over-estimation of the reference pressure respectively. Three monitors showed consistent negative bias, with every measurement being underestimated with respect to the reference pressure recorded by the Omron PA-350. 
Measurements from two monitors - the Boots Clinically Validated Arm Monitor (BCV), and the Alvita MC101 were compared to those from the Homedics BPA-300 in 21 adult volunteers. Participants were predominantly female ( $86 \%$ female, $14 \%$ male), with an average age of 40.8 years (range 25-61). Compared to the reference monitor, the BCV showed a median (IQR) systolic error of $6 \mathrm{mmHg}$ ( 2 to $10 \mathrm{mmHg}$ ) and diastolic error of $-3 \mathrm{mmHg}(-6$ to $0 \mathrm{mmHg}$ ) and the Alvita MC101 showed a median (IQR) systolic error of $2 \mathrm{mmHg}$ (2 to $8 \mathrm{mmHg}$ ) and median diastolic error of $-3 \mathrm{mmHg}(-6$ to $1 \mathrm{mmHg})$.

\section{Discussion}

Monitors available for sale to the general public were generally accurate in measuring static pressure, with a lack of correlation between monitor retail price and accuracy in measuring static pressure. However, in vivo analysis of two monitors produced greater errors in the measurement of systolic and diastolic pressure than was seen during the static pressure testing. This suggests that static pressure testing may not be an appropriate way of investigating monitor accuracy.

Our study contains a number of limitations. Firstly, static pressure testing seems to be a poor indicator of monitor accuracy in vivo. As such, any of the conclusions we have made based on static pressure results remain to be validated in vivo. The fact that a small number of monitors were incompatible with static pressure testing also limits this method as a way of investigating monitor accuracy. Our study was limited to one device for each of the models tested. We assume that variation within models will be minimal, as devices should be mechanically identical and contain the same programming, but testing to confirm this would be a useful next step. Although we purchased a broad range of different blood pressure monitor models, our sample was limited to models that were on sale in Oxford pharmacies during the study period, and is not comprehensive. We have also not evaluated differences between devices which measure blood pressure at the wrist and those measuring it on the upper arm. 
The in vivo testing was limited by the small number of monitors which were investigated. Therefore, we must be cautious in making any generalised statements on the accuracy of monitors in systolic and diastolic testing compared to static pressure testing. The reference standard for our in vivo testing was the Homedics BPA-300 digital monitor. This monitor performed well compared to other validated monitors in a published systematic review,[4] but is not equivalent to using a mercury sphygmomanometer, which is considered the gold standard by international validation protocols. Thus, our results cannot be considered equivalent to a validation study, and would require further confirmation by comparing static tests to full compliant validation.

In summary, this pilot study showed that monitors bought in pharmacies were surprisingly accurate in the measuring of static pressure, but displayed far less accuracy in vivo. The disparity in these results indicate that static pressure testing alone may not be sufficient to assess the accuracy of blood pressure monitors on sale to the public. 


\section{References}

1 O'Brien E, Petrie J, Littler W, de Swiet M, Padfield PL, Altman DG et al. An outline of the revised British Hypertensive Society protocol for the evaluation of blood pressure measuring devices. J Hypertens 1993; 11(6): 677-679

2 Association for the Advancement of Medical Instrumentation. American National Standard. Manual, electronic or automated sphygmomanometers ANSI/AAMI: Arlington, VA USA, 2003

3 O'Brien E, Pickering T, Asmar R, Myers M, Parati G, Staessen J, et al. Working Group on Blood Pressure Monitoring of the European Society of Hypertension International Protocol for validation of blood pressure measuring devices in adults. Blood Press Monit 2002; 7(1): 317

4 Wan Y, Heneghan C, Stevens R, McManus RJ, Ward A, Perera R et al. Determining which automatic digital blood pressure device performs adequately: a systematic review. J Hum Hypertens 2010; 24: 431-438

5 A'Court C, Sanders S, Ward A, McManus R, Heneghan C. Type and accuracy of sphygmomanometers in primary care: a cross-sectional observational study. BJGP 2011; 61(590): e 598-603 


\section{Figure Caption}

Figure 1: Box plot showing median (central line), IQR (box) and range (dotted lines) of errors for tested monitors, listed in price order 